\title{
Reconsideración del discurso interactivo en la clase de L2 o LE
}

\section{Miquel Llobera}
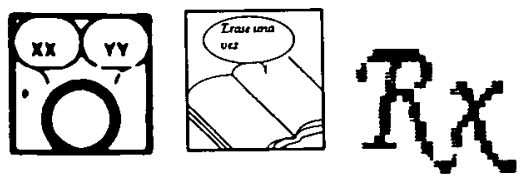

El autor reivindica el papel didáctico del discurso en el aula. Desde su punto de vista, en los últimos años, se ha enfatizado en demasía la presentación de materiales didácticos "auténticos" en detrimento de considerar como "auténtico» el propio discurso producido en el aula. De hecho, el autor considera que, más allá de ser considerado como input comprensible, el discurso producido en el aula ba tenido una relevancia poco importante $y$, por eso, en el marco de la construcción de un discurso significativo en la relación profesor-alumno como base del progreso docente, el papel didáctico del discurso en el aula pasa a ocupar un lugar principal.

\section{INTRODUCCION}

En el inicio de la década que acabamos de dejar hubo un slogan muy popular entre profesores de lengua extranjera (LE, desde aquí) que traducido sería algo así como "dejad entrar el mundo real en la clase de LE». Fue el momento de los documentos reales, de los materiales de comprensión oral grabados en vivo, sin usar locutores o actores en las salas de grabación, como se había hecho hasta este momento. Este slogan se tradujo en el hecho de que muchos libros de texto presentasen reproducciones de materiales escritos que inicialmente no habían sido elaborados con finalidad didáctica, y que tenían que llegar a resultar comprensibles a través de procesos didácticos que excluyeran la traducción. Este afán de realismo estaba avalado por toda una serie de hipótesis sobre la manera en que se producía la adquisición, y su aportación fue positiva porque introdujo un cierto rigor al intentar definir las características del discurso escrito y evitó alguna de aquellas producciones pedagógicas tan artificiosas y que habían sido tan duramente criticadas (Widdowson, 1984: 162-169). 


\section{EL DISCURSO PRODUCIDO Y EL DISCURSO APORTADO, EN EL AULA DE LE}

Este interés centrado en los discursos orales y escritos que se aportaban a las clases impidió que se prestase suficiente atención a los discursos producidos en la misma clase excepto en un sentido: el discurso interactivo del profesor-alumnos o el de los alumnos entre sí como input comprensible. Se daba así una extraña situación en la que los investigadores de la realidad docente se interesaban por el discurso "producido" mientras que los metodólogos y los productores de materiales se interesaban sobre todo por la «autenticidad» de los discursos «aportados» dificultando de esta manera el necesario diálogo para avanzar con fundamento hacia una enseñanza de LE que superase simplificaciones y maniqueísmos. Dejando pues aparte los estudios muy importantes de Adquisición de Segunda Lengua, que despertaron el interés de los docentes mejor informados, la interrelación lingüística profesor-estudiantes de LE fue poco considerada: como si fuese un subproducto inevitable, la ganga que permitía pasar de una actividad significativa a otra, o un subproducto que regulaba la vida de la clase pero que carecía de funcionalidad didáctica propia, es decir que no podía contribuir al aprendizaje de la LE.

Sin embargo una aproximación con mayor detalle a este discurso permite plantear una serie de hipótesis que va en sentido contrario:

1. El discurso en el aula, a pesar de sus peculiaridades, presenta muchas de las características que presenta el discurso en las interacciones sociales generales.

2. Es la exposición de estas características lo que permite al alumno construir sus hipótesis sobre las reglas de uso del discurso, la aplicación de las cuales le permitirá comunicarse (Kasper, 1989).

3. Es la actuación en la interacción lo que permite la construcción de un discurso significativo en la clase.

4. La construcción de un discurso significativo parece ser condición básica para el aprendizaje. Aun cuando el significado de este discurso tenga la peculiaridad de adquirir sentido sólo a través de su finalidad docente.

\section{DISCURSO PRODUCIDO EN CLASE DE LE Y DISCURSO ORDINARIO}

Veamos pues la primera hipótesis que considera que el discurso oral que se da en la clase de LE contiene muchas características del discurso ordinario de la LE que se estudia.

Es cierto que en los trabajos publicados sobre el lenguaje que se da en clase, éste se presenta como un discurso muy particular, en el que a una respuesta de uno de los interlocutores, quien ha iniciado la interacción responda con una evaluación:

Ejemplo: P.: «¿Qué hora es?»

E.: «Las tres y cuarto.»

P.: «iMuy bien!» 
Cuando lo normal, fuera del aula, hubiera sido contestar con una expresión de agradecimiento como "gracias» o con un comentario: «jSe me está haciendo tarde!», etc. Para más detalle sobre este movimiento de iniciación-respuesta-evaluación ver los trabajos de Sinclair y Coulthard (1975) o los de Tsui-Bik-May (1987), aplicando el mismo método de análisis.

Otros autores han subrayado el hecho que las preguntas del profesor en las clases de LE están mucho más pendientes de conseguir de sus interlocutores expresiones formalmente correctas que expresiones con información exacta, hasta el punto que, como dicen Long y Sato (1983), «la conversación entre hablantes nativos y hablantes no nativos en las clases de segundas lenguas se presenta como una versión distorsionada de la que da en el mundo real».

Finalmente, también se ha puesto de relieve el carácter extraño del discurso de clase de LE, al caracterizarlo como "discurso teatralizado", con el contenido transformado en «forma a estudiar», o desviado de su uso común: «Los discursos producidos en clase, debido a que son discursos destinados al aprendizaje de una lengua, son a menudo "subvertidos", extraídos de su circuito comunicativo originario, cambiando de esta manera de status lingüístico" (Cicourel, 1986).

Sin embargo, al subrayar las diferencias entre discurso en la clase de LE del discurso ordinario se ha obviado la gran cantidad de coincidencias existente entre ambos. Quizá esta falta de percepción venga dada por influencia de la práctica dominante durante los enfoques estructuralistas en los que el discurso en la clase debía estar totalmente controlado. Hoy en día, aparte de la comprensibilidad, se exigen pocas condiciones de control de dificultad y secuenciación.

Veamos un fragmento de una clase desde la óptica de los elementos comunes existentes entre el discurso de la clase LE y el discurso del intercambio ordinario. Para estudiar este fragmento nos basaremos en la distribución de turnos y comentaremos algunos de los contenidos:

1. P.: «El otro día teníamos dos preguntas. Una pregunta es ¿qué hacía Amanda normalmente?... ¿Alguien puede explicar qué hacía Amanda normalmente? ¿Cómo se sentía?»

2. E.: (Respuesta audible, pero incomprensible) (Risa).

3. P.: «¿Puede haber más información, no?, ¿dónde?, ¿cuándo?, ¿por qué?»

4. E.: (Respuesta incomprensible).

5. P.: «Iba a la fábrica a decirle, ¡HOLA!»

6. E.: «iNo!» (Risa).

7. E.: (Incomprensible, intervención muy breve, sonrisa).

8. P.: (Risa), «¿En qué momento del día? ¿Por qué iba a la fábrica...?

9. A.: « ¿Cuando había un descanso!»

10. P.: «iAjá!»

En esta transcripción de un vídeo de una clase de Español como LE se pueden percibir varios elementos que son comunes en la conversación fuera del aula.

Si nos centramos en la distribución y regulación de los turnos de habla tal como fueron definidos originalmente por Sacks, Schegloff y Jefferson (1974), nos damos cuenta de que algunas de las características que ellos ob- 
servaban en las conversaciones ordinarias se dan en este fragmento de una transcripción de clase:

a) Quizá lo más sorprendente sea el parcial cumplimiento del punto 5 del trabajo antes citado que decía literalmente «Turn order is not fixed, but varies" (1974: 699). En este caso, naturalmente, lo que varía es el orden de intervención de los alumnos puesto que ninguno de ellos es designado de antemano, a diferencia del profesor $y$, como ocurre en conversaciones donde el discurso refleja una jerarquía socialmente aceptada, el profesor tiene derecho permanente a turno. Esta falta de fijación de los turnos ayuda a mantener la «intrinsic motivation for listening» (op. cit., 727), que es uno de los rasgos de la conversación ordinaria.

b) Los hablantes mantienen turnos breves, propios de los intercambios lingüísticos ordinarios; aún cuando se cumple el puntos seis del mismo trabajo según el cual «Turn size is not fixed but varies».

c) P y E alternan sus contribuciones de manera ordenada, excepto en el caso de E2 que en cierto modo "roba" (ver Allwright, 1980: 168 y 172) un turno.

d) Esta apropiación no ha sido precedida de señales de querer el turno, y por su brevedad se interpreta como una contribución marginal que no interfiere en la distribución de turnos de intervención que acaba de darse.

e) La intervención (Int., desde ahora) de E.2 también refleja una situación en la que la jerarquía profesor-alumno está mitigada por la actitud de la profesora, por la edad de los participantes, todos los alumnos son adultos y varios de ellos son más viejos que $\mathrm{P}$. y por las normas de conducta de la sociedad, en la que un cierto encabalgamiento en la distribución de turnos es considerado como normal. Es un ejemplo más de lo que Van Lier (1988: 133) define como opinión generalmente admitida entre los analistas de la conversación de que "exista un sistema de interacción básico y no marcado que es el de la conversación general", haciéndose eco del punto de vista de Sacks et al. (1974), según el cual (la) «conversación debería ser considerada como la forma básica del sistema de intercambio lingüístico y por tanto que el discurso de la clase de LE presenta un carácter derivativo del general $\mathbf{u}$ ordinario.

\section{COMPETENCIA PRAGMATICA E IL}

En relación con la segunda hipótesis la evidencia no será tan explícita (para estudios más específicos de pragmática en Interlenguaje, ver G. Kasper, 1989), aunque si nos fijamos en aspectos más cualitativos es evidente que nos encontramos ante una serie de fenómenos que damos por sentados pero que bastaría con ejercitar un poco la memoria para darnos cuenta que son un producto de una situación de intercambio lingüístico en un momento determinado y en una sociedad determinada:

a) El humor es aceptable en situaciones donde la jerarquía de los hablantes es diferente ( $\mathrm{Ej}$. Int. n. ${ }^{\circ}$ e Int. n. ${ }^{\circ} 5$ ), aun cuando en ésta 
el humor tiene la función de mostrar mediante la ironía, la posición de poder de la profesora y la utilización didáctica de la misma.

b) La contribución del hablante puede ser breve y no tiene por qué mostrar ninguna característica de deferencia del inferior al superior. (Ej.: Int. n. ${ }^{\circ} 6$ ).

c) El alumno puede responder a preguntas del tipo «display questions» con respuestas en las que no debe exhibirse la oración completa, sino la parte con la que se responde en el discurso ordinario $(\mathrm{Ej}$.: Int. n. ${ }^{9}$ 9). Para darnos cuenta de este hecho basta contrastar con las respuestas exigidas durante muchos años en las clases y que en este caso hubiera sido «Manuel iba a la fábrica cuando había un descanso".

d) El hablante en una posición jerárquicamente inferior no siempre tiene que dejar al otro interlocutor acabar con su reformulación tal como aparece en Int. 8 e Int. 9.

Hay otros elementos que conviene destacar. Uno de ellos es la abundancia de reformulación que puede parecer sólo típica de la clase de LE, pero basta estudiar transcripciones de diálogos entre hablantes nativos y hablantes no nativos para darse cuenta de la frecuencia de la reformulación en este tipo de intercambios lingüísticos. Aunque es cierto que parece poco probable que las reformulaciones en el discurso ordinario presenten características tan profesorales como Int. 3 pero no parece imposible que se den reformulaciones como en Int. 8. Como también parece plausible que fuera del aula, entre un hablante nativo y un hablante no nativo se diese una intervención como ésta, en una conversación sobre medidas para disminuir la polución atmosférica:

P.: «Another thing you can do is keep your car, your car, tuned. Your car in good condition. Send it to the garage every once in a while to have it in very good condition. So it won't produce excess of fumes,... too much"

(Otra cosa que se puede hacer es mantener su coche, su coche, bien ajustado. Su coche en buen estado. Lo envías al garage de vez encuando para que esté en buen estado. Así no producirá esceso de humos,... demasiados.)

El hablante nativo teme que el interlocutor no entienda la palabra «tuned» y por ello la reformula como «in good condition» o reformula «excess» como «too much». De la misma manera que un hablante no nativo para hacerse comprender de un nativo reformula la palabra pronunciada como «chawch..." mediante una paráfrasis «like American people go there every Sunday, you know... the American people get dressed up to go to um Chwach» (Pica, 1988).

Sería inexacto no señalar algunas de las discrepancias mayores que se encuentran entre la "conversación» en la clase con los rasgos de la conversación general según el modelo de Sacks et al. (1974). Así estos autores en los puntos siete y ocho de su sistemática afirman que «legth of conversation is not fixed in advance" $y$ "what parties say is not fixed in advance». Naturalmente la conversación en la clase no puede exceder el tiempo fijado por el horario institucionalizado y el tema que se va a tratar está fijado por el syllabus y por las afirmaciones iniciales del profesor, así como por las actividades didácticas que preceden este fragmento. Estas diferencias no tie- 
nen que ocultar un hecho básico que es común al discurso de clase y el discurso en general, y éste es el aspecto de construcción conjunta entre los interlocutores que intervienen. Es decir que el progreso de la interacción didáctica está tan condicionada en cuanto a su desarrollo al hecho de que los interlocutores hagan las aportaciones que se espera de ellos como lo está el desarrollo de la conversación ordinaria. Si el profesor no obtiene respuesta a sus invitaciones a intervenir o en el caso de clases con la atención muy centrada en la actuación del profesor, si el profesor no obtiene la respuesta mínima considerada como aceptable la clase se convierte en una operación complicada: bastaría imaginar una clase donde los alumnos no atendieran; es decir, no asintiesen con la cabeza, no tomasen notas, no mirasen hacia el profesor, sino que mantuviesen una mirada impávida hacia el vacío para percibir qué mínimo de interacción es indispensable para que una clase tenga lugar. $Y$ con estas consideraciones nos hemos metido de lleno en las hipótesis tres y cuatro que nos planteábamos al principio de este argumento.

Para cerrar el argumento de este trabajo conviene volver un poco a las consideraciones que hacíamos sobre la docencia al principio de este trabajo y veamos qué repercusiones tiene en la didáctica de LE esta reconsideración de los discursos que se dan en la clase de LE.

Al final del estructuralismo metodológico (Metodología SGAV, por ejemplo), los modelos de lengua aportados a clase fueron vistos como insuficientes, por su artificialidad, y se puso énfasis en la aportación de realia. Los primeros materiales discursivos -es decir que tomaban en cuenta los actos de habla como elementos mínimos de la lengua - liberados de los rigores de la secuenciación estructural presentaban un grado de plausibilidad mayor. Como ejemplo, comparar Abbs (1975) con Alexander (1973), para mencionar dos autores de una misma editorial. Pero ni en uno ni otra aproximación se contemplaba el discurso producido en clase como material de instrucción. Es decir, que aún en este momento el énfasis estaba en el «usage», según la distinción de Widdowson, y no en el «use». Es decir que en muchas aulas todavía se "promovía la habilidad de manifestar la lengua como utilización ("usage") antes que el llevar a cabo comunicaciones genuinas que se realizan en el uso ("use") de la misma», Widdowson (1988). En los últimos diez años el cambio de perspectiva se ha consolidado. La lengua en clase no sólo cumple el parámetro de gramaticalidad —con ciertas limitaciones propias del Interlenguaje- de Hymes (1972), sino que además tiene tendencia a respetar el de adecuación e incluso el de ocurrencia -es decir, que se dé en la realidad. Y este último parámetro se da sobre todo cuando se integra el discurso producido en la clase en el contenido instruccional.

\section{CONCLUSIONES}

El cambio paulatino de consideración del discurso producido en el aula quizá sea el cambio más importante que se está produciendo en estos momentos en todas las tendencias innovadoras de la enseñanza de LE. Y sin este cambio de consideración no es posible situar adecuadamente la promoción de las estrategias humanistas, ni el énfasis en desarrollar la autonomía del «aprendiente» mediante estrategias de aprendizaje y comunicativas, 
y ni siquiera las aproximaciones curriculares procesuales. Este cambio me parece que entra de lleno en el centro de la práctica de la enseñanza de la LE hasta tal punto que me parece que no podría argumentar que de una manera paulatina se ha ido pasando de una aproximación estructural a una aproximación discursiva.

Es por ello que la distinción entre discurso aportado y discurso producido en la clase de LE puede ser visto como contextos que se integran y producen un discurso significativo, en un sentido muy específico (Green et al., 1988: 11-47). Este discurso tiene sentido en cuanto llevan a cabo felizmente su función didáctica, y ésta se convierte en su contenido genuino.

Parece que una adecuada comprensión de la naturaleza de este discurso comporta un volumen de investigación que este trabajo sólo ha podido apuntar para poder disponer de información específica cuando se toman decisiones de innovación curricular, para poder superar las consabidas generalizaciones que nada aportan en lo que se refiere a la práctica en el aula de LE.

\section{Referencias}

ABBS et al. (1975). Strategies, Londres, Longman.

ALEXANDER, L. G. (1973). Mainline progress $A$, Londres, Longman.

ALLWRIGHT, R. (1980). «Turns topics and tasks: patterns of participation in language learning and teaching». En Larsen-Freeman (ed.), Discourse Analisis in Second Language, Rowley Mass., Newbury House.

CicOuREL, F. (1984). *La construction dé l'interaction didactique», ELA, vol. 55, 47-57.

Gass, S., Madden, C., Preston, D. y Selinker, L. (eds.) (1989). Variation in second language acquisition: discourse and pragmatics, Clevendon, Multilingual Matters.

GREEN, J. L. y HARKER, J. L. (eds.) (1988). Multiple perspective analyses of classroom discourse, Norwood, NJ, Ablex.

HYMES, D. (1972). «On communicative competence». En J. P. Prida y J. Holmes (eds.), Sociolinguistics, Hardmondsworth, Penguin.

KASPER, G. (1989). «Variation in interlanguage speach act realization». En S. Gass et al. (eds.) (1989), Variation in second language acquisition: discourse and pragmatics, Clevendon, Multilingual Matters.

LIER, L. VAN (1988). The classroom and the language learner, Londres, Longman.

LONG, M. y SATO, S. (1983). *Classroom foreign talk discourse: forms and punctions of teachers questions». En H.W. Seliger y M. H. Long (eds.) (1983), Classroom oriented research in second language acquisition, Rowley, Massachusetts, Nowbury House, 268-285.

PICA, T. (1988). «Interlanguage adjustments as an outcome of NS-NNS negotiated interaction, Language Learning, vol. 38, 1, 45-73.

SACKS, H., SCHEGLOFF, G. y JeFFERSON, G. (1974). *A simplest systematic for organisation on turn-talking for conversation», Languaje, vol. 50.4, 697-735.

SinCLAIR, J. MCH. y COULTARD, M. (1975). Towards an analysis of discourse, Oxford, Oxford University Press.

TsUY-BIK-MAY, A. (1987). *An analysis of different types of interaction in ESL classroom discourse», IRAL, vol. XXV, 4, 336-353.

WIDDOwsON, H. G. (1984). "The use of literature". En Explotarions in applied linguistics, Oxford, Oxford University Press, 160-173.

WidDowson, H. G. (1989). «Knowledge of language and ability for usem. En Applied Linguistics, vol. 10, 2, 128-131. 
Reconsideración del discurso interactivo en la clase L2 o LE. M. Llobera.

CLEE, 1990, 7-8, pp. 91-98.

Resumen: Este artículo presenta el discurso en clase de LE como un discurso auténtico que tiene una finalidad precisa y especifica, que no puede ser reducido a mero modelo de las formas de la lengua o a mero suministro de input adecuado para que el aprendiente de $L E$ desarrolle su lenguaje, y argumenta que es importante desarrollar una mejor percepción del mismo para poder hablar de innovaciones en los syllabi o de cambio de paradigma, mediante una serie de análisis de muestras de discurso en el aula de LE.

Datos sobre el autor: Miquel Llobera es Catedrático E.U. en el Departamento de Didáctica de la Lengua, Universidad de Barcelona. Dirige el Master de Formación de Profesores de Lengua Extranjera (tanto de español como lengua extranjera como de inglés). Fue el asesor didáctico y coautor del libro del profesor del multimedia para el catalán como L2, Digui, Digui (1984-1986), Publicacions de l'Abadia de Montserrat, Barcelona. Es coautor de los manuales para la enseñanza del inglés Open Line (1987, 1988, 1989), Alhambra-Longman, Madrid. Le interesa la dimensión educativa de la enseñanza de LE, el discurso que surge en este tipo de docencia y las narrativas que subyacen en esta práctica.

Dirección: Escola d'Idiomes Modernes. Gran Via de les Corts Catalans, 585. 08007 Barcelona.

(C) De todos los artículos deberá solicitarse por escritor autorización de CL\&E y de los autores para el uso en forma de facsímil, fotocopia o cualquier otro medio de reproducción impresa. CL\&E se reserva el derecho de interponer las acciones legales necesarias en aquellos casos en que se contravenga la ley de derechos de autor. 